\title{
WHICH AMALGAMS ARE CONVOLUTION ALGEBRAS?
}

\author{
JAMES STEWART $^{1}$ AND SALEEM WATSON
}

\begin{abstract}
We determine necessary and sufficient conditions on a locally compact abelian group $G$ for the amalgam $\left(L^{p}, l^{4}\right)(G)$ to be an algebra under convolution. If $q>1, G$ must be compact; if $p<1, G$ must be discrete. If $p \geqslant 1$ and $q \leqslant 1$, the amalgam is always an algebra.
\end{abstract}

1. Introduction. The amalgam of $L^{p}$ and $l^{q}$ is the space $\left(L^{p}, l^{q}\right)(G)$ consisting of all functions on a locally compact abelian group $G$ which are locally in $L^{p}$ and have $l^{q}$ behavior at infinity in the sense that the $L^{p}$-norms over certain compact subsets of $G$ form an $l^{q}$-sequence. (Precise definitions will be given in the next section.) We pose and answer the question: "Which of the amalgam spaces $\left(L^{p}, l^{q}\right)(G)$ are algebras under convolution?".

If $p=q$, then the amalgam $\left(L^{p}, l^{q}\right)(G)$ reduces to $L^{p}(G)$. For this case it is well known that $L^{1}(G)$ is always an algebra, and for $p>1$ Żelazko [11] has proved that $L^{p}(G)$ is an algebra if and only if $G$ is compact. For the amalgams $\left(L^{p}, l^{q}\right)(G)$ with $p \geqslant 1, q \geqslant 1$, it is known that $\left(L^{p}, l^{1}\right)(G)$ is always an algebra, and we prove here that $\left(L^{p}, l^{q}\right)(G), q>1$, is an algebra if and only if $G$ is compact.

For indices smaller than 1 , Żelazko [12] has shown that $L^{p}(G), 0<p<1$, is an algebra if and only if $G$ is discrete. Likewise, for amalgams, it turns out that if $\left(L^{p}, l^{q}\right)(G)$ is an algebra for $0<p<1$, then $G$ is discrete.

However, we also show that if $p \geqslant 1$ and $0<q \leqslant 1$, then $\left(L^{p}, l^{q}\right)(G)$ is always an algebra. This provides a large class of new convolution algebras which, for $q<1$, are $F$-algebras.

2. Amalgams. For functions of a real variable, Holland [4] defined the amalgam of $L^{p}$ and $l^{q}$ as the space $\left(L^{p}, l^{q}\right)$ of functions $f$ such that

$$
\|f\|_{p, q}=\left[\sum_{n=-\infty}^{\infty}\left[\int_{n}^{n+1}|f(x)|^{p} d x\right]^{q / p}\right]^{1 / q}<\infty
$$

although certain special cases had been studied earlier starting with Wiener [10]. For functions on a locally compact abelian group $G$, amalgams have been defined and studied by Bertrandias, Datry and Dupuis [1], Stewart [8], and Busby and Smith [2]. In particular we follow the approach of [8] by using the structure theorem to write

\footnotetext{
Received by the editors March 6, 1984. Presented January 10, 1985 at the annual meeting of the American Mathematical Society, Anaheim, California.

1980 Mathematics Subject Classification. Primary 43A15, 43A20.

Key words and phrases. Amalgam, convolution algebra.

${ }^{1}$ This work was supported by NSERC grant A4833.
} 
$G=R^{a} \times G_{1}$, where $a$ is a nonnegative integer and $G_{1}$ is a group which contains a compact open subgroup $H$. The Haar measure $m$ on $G_{1}$ is normalized so that $m(H)=1$. Define $I=[0,1)^{a} \times H$ and $I_{\alpha}=g_{\alpha}+I$, where each $g_{\alpha}$ is of the form $\left(n_{1}, \ldots, n_{a}, t\right)$ with $n_{i} \in Z$ and the $t$ 's being a transversal of $H$ in $G_{1}$, that is, $G_{1}=$ $\bigcup_{t}(t+H)$. We can then write $G$ as a disjoint union:

$$
G=\bigcup_{\alpha \in J} I_{\alpha}
$$

In terms of this decomposition we define the amalgam $\left(L^{p}, l^{q}\right)(G)$ to be the space of functions $f$ which are locally in $L^{p}$ and are such that

$$
\|f\|_{p, q}=\left[\sum_{\alpha \in J}\left[\int_{I_{\alpha}}|f(x)|^{p} d x\right]^{q / p}\right]^{1 / q}<\infty .
$$

For $p=\infty$ we have

$$
\|f\|_{\infty, q}=\left[\sum_{\alpha \in J} \sup _{x \in I_{\alpha}}|f(x)|^{q}\right]^{1 / q}<\infty \quad(q<\infty) .
$$

We shall often use the notation $f_{\alpha}$ to mean the function which agrees with $f$ on $I_{\alpha}$ and is 0 elsewhere. Then we can write

$$
\|f\|_{p, q}=\left[\sum_{\alpha \in J}\left\|f_{\alpha}\right\|_{p}^{q}\right]^{1 / q}
$$

Notice that if $G$ is compact, then $\left(L^{p}, l^{q}\right)(G)=L^{p}(G)$. If $G$ is discrete, then we can take $I=\{0\}$ and so $\left(L^{p}, l^{q}\right)(G)=l^{q}(G)$.

Previously, amalgams have been studied for $p \geqslant 1, q \geqslant 1$, and in this case it is known that $\|f\|_{p . q}$ is a norm and $\left(L^{p}, l^{q}\right)(G)$ is a Banach space with dual $\left(L^{p^{\prime}}, l^{q^{\prime}}\right)(G)$, where $1 / p+1 / p^{\prime}=1,1 \leqslant p, q<\infty$ [1]. However, if either $p$ or $q$ is less than 1 , then $\left(L^{p}, l^{q}\right)$ is an $F$-space. To show this we establish that $\|f\|_{p, q}$ is a quasinorm by using the following inequalities [5, p. 158]:

$$
\begin{gathered}
(a+b)^{p} \leqslant 2^{p-1}\left(a^{p}+b^{p}\right) \quad(p>1), \\
(a+b)^{p} \leqslant a^{p}+b^{p} \quad(0<p<1), \\
\|f+g\|_{p} \leqslant 2^{(1-p) / p}\left(\|f\|_{p}+\|g\|_{p}\right) \quad(0<p<1) .
\end{gathered}
$$

For $p \geqslant 1, q<1$, we have

$$
\begin{aligned}
\|f+g\|_{p, q}^{q} & =\sum_{\alpha \in J}\left\|(f+g)_{\alpha}\right\|_{p}^{q} \leqslant \sum_{\alpha \in J}\left(\left\|f_{\alpha}\right\|_{p}+\left\|g_{\alpha}\right\|_{p}\right)^{q} \\
& \leqslant \sum_{\alpha \in J}\left\|f_{\alpha}\right\|_{p}^{q}+\sum_{\alpha \in J}\left\|g_{\alpha}\right\|_{p}^{q}[\text { by }(2.2)] \\
& =\|f\|_{p, q}^{q}+\|g\|_{p, q}^{q} .
\end{aligned}
$$

Then (2.1) gives

$$
\|f+g\|_{p, q} \leqslant 2^{(1-q) / q}\left(\|f\|_{p, q}+\|g\|_{p, q}\right) \quad(p \geqslant 1, q<1) .
$$


Similarly, using (2.1), (2.2) and (2.3) we obtain

$$
\begin{gathered}
\|f+g\|_{p, q} \leqslant 2^{(1-p) / p}\left(\|f\|_{p, q}+\|g\|_{p, q}\right) \quad(p<1, q \geqslant 1), \\
\|f+g\|_{p, q} \leqslant 2^{(1-p) / p} 2^{(1-q) / q}\left(\|f\|_{p, q}+\|g\|_{p, q}\right) \quad(p<1, q<1) .
\end{gathered}
$$

The inequalities (2.4), (2.5) and (2.6) show that when either $p$ or $q$ is less than 1 , $\left(L^{p}, l^{q}\right)(G)$ is a quasi-normed space which is therefore locally bounded [5, p. 159] and hence an $F$-space [7].

The following relations were given in [8] for $p, q \geqslant 1$ but continue to hold when either index is less than 1 :

$$
\begin{array}{ll}
\left(L^{p}, l^{q_{1}}\right) \subset\left(L^{p}, l^{q_{2}}\right) & \left(0<p \leqslant \infty, 0<q_{1} \leqslant q_{2} \leqslant \infty\right), \\
\left(L^{p_{2}}, l^{q}\right) \subset\left(L^{p_{1}}, l^{q}\right) & \left(0<p_{1} \leqslant p_{2} \leqslant \infty, 0<q \leqslant \infty\right), \\
\left(L^{p}, l^{p}\right)=L^{p} & (0<p \leqslant \infty), \\
\left(L^{p}, l^{q}\right) \subset L^{p} \cap L^{q} & (0<q \leqslant p \leqslant \infty), \\
L^{p} \cup L^{q} \subset\left(L^{p}, l^{q}\right) & (0<p \leqslant q \leqslant \infty) .
\end{array}
$$

Associated with (2.7) and (2.8) are the inequalities

$$
\begin{array}{ll}
\|f\|_{p, q_{2}} \leqslant\|f\|_{p, q_{1}} & \left(0<p \leqslant \infty, 0<q_{1} \leqslant q_{2} \leqslant \infty\right), \\
\|f\|_{p_{1}, q} \leqslant\|f\|_{p_{2}, q} & \left(0<p_{1} \leqslant p_{2} \leqslant \infty, 0<q \leqslant \infty\right) .
\end{array}
$$

3. The case $p \geqslant 1, q \geqslant 1$. If we combine Young's Inequality for amalgams [ 1 or 2] with the inequality (2.13), we get

$$
\|f * g\|_{p, 1} \leqslant C\|f\|_{1}\|g\|_{p, 1} \leqslant C\|f\|_{p, 1}\|g\|_{p, 1},
$$

and this shows that $\left(L^{p}, l^{1}\right)(G)$ is a Banach algebra for $p \geqslant 1$ and for any $G$. (In fact, it is a Segal algebra $[6,1]$. The classic special case is the Wiener algebra $\left(L^{\infty}, l^{1}\right) \cap C(R)$ as studied by Goldberg [3].)

We now consider the case $p \geqslant 1, q>1$. Here $\left(L^{p}, l^{q}\right)(G)$ is a Banach space, so if it is a topological algebra, then it is well known that there is an equivalent submultiplicative norm $\|\cdot\|$ such that $\|f\| \geqslant\|f\|_{p, q}$. The proof of the following theorem is adapted from Urbanik [9].

THEOREM 1. The amalgam $\left(L^{p}, l^{q}\right)(G), p \geqslant 1, q>1$, is a topological algebra if and only if $G$ is compact.

Proof. If $G$ is compact, then $\left(L^{p}, l^{q}\right)(G)=L^{p}(G)$ which is known to be an algebra [11].

Now suppose that $A=\left(L^{p}, l^{q}\right)(G)$ is a topological algebra. We first show that $A \neq \operatorname{rad}(A)$. Let $V$ be a symmetric, compact neighborhood of 0 in $G$ and let $\chi$ be the characteristic function of $V+V$. Then, for $x \in V$, we have

$$
\chi^{n+1}(x)=\int_{G} \chi(x-y) \chi^{n}(y) d y \geqslant \int_{V} \chi^{n}(y) d y
$$


where $\chi^{n}$ means the $n$-fold convolution of $\chi$. By induction it follows that $\chi^{n+1}(x) \geqslant$ $[m(V)]^{n}$ when $x \in V$. Putting $c=1 /\|\chi\|$, we obtain

$$
\begin{aligned}
\left\|\chi^{n}\right\| & \geqslant c\left\|\chi^{n+1}\right\| \geqslant c\left\|\chi^{n+1}\right\|_{p, q} \\
& =c\left[\sum_{\alpha \in J}\left[\int_{I_{\alpha}}\left|\chi^{n+1}\right|^{p}\right]^{q / p}\right]^{1 / q} \geqslant c\left[\sum_{\alpha}\left[\int_{I_{\alpha} \cap V}\left|\chi^{n+1}\right|^{p}\right]^{q / p}\right]^{1 / q} \\
& \geqslant c\left[\sum_{\alpha}\left[\int_{I_{\alpha} \cap V}[m(V)]^{n p}\right]^{q / p}\right]^{1 / q}=c\left[\sum_{\alpha} m(V)^{n q} m\left(V \cap I_{\alpha}\right)^{q / p}\right]^{1 / q} \\
& \geqslant c[m(V)]^{n}\left[\sum_{\alpha}\left[m\left(V \cap I_{\alpha}\right)\right]^{q / p}\right]^{1 / q}>0 .
\end{aligned}
$$

Thus

$$
\lim _{n \rightarrow \infty}\left\|\chi^{n}\right\|^{1 / n} \geqslant m(V)>0
$$

and so $\chi \notin \operatorname{Rad}(A)$. Therefore there is a nontrivial multiplicative linear functional $T$ on $A$. Since $T$ is continuous there exists a function $f$ in $\left(L^{p^{\prime}}, l^{q \prime}\right)$ with $\|f\|_{p^{\prime}, q^{\prime}}>0$ such that

$$
T(\phi)=\int_{G} f(x) \phi(x) d x \quad\left(\phi \in\left(L^{p}, l^{q}\right)\right) .
$$

Since $T$ is multiplicative, for every $\phi, \psi \in\left(L^{p}, l^{q}\right)$ we have

$$
\begin{aligned}
\int_{G} \int_{G} f(x+y) \phi(x) \psi(y) d x d y & =\int_{G} f(x)\left[\int_{G} \phi(x-y) \psi(y) d y\right] d x \\
& =\int_{G} f(x)(\phi * \psi)(x) d x=T(\phi * \psi)=T(\phi) T(\psi) \\
& =\int_{G} \int_{G} f(x) f(y) \phi(x) \psi(y) d x d y .
\end{aligned}
$$

This shows that

$$
f(x+y)=f(x) f(y) \quad \text { (l. a.e. }) .
$$

Now we use the fact that $\left(L^{p}, l^{q}\right)$ has an equivalent translation-invariant norm $\|\cdot\|_{p, q}^{\#}[1$, Proposition VIII]:

$$
c^{\prime \prime}\|f\|_{p, q}^{\#} \leqslant\|f\|_{p, q} \leqslant c^{\prime}\|f\| p_{p, q}^{\#} .
$$

It follows that translation is a bounded operator on $\left(L^{p}, l^{q}\right)$ :

$$
\left\|f_{y}\right\|_{p, q} \leqslant K\|f\|_{p, q}
$$

where $f_{y}(x)=f(x+y)$. Applying this to $\left(L^{p^{\prime}}, l^{q \prime}\right)$, we have

$$
\begin{aligned}
\|f\|_{p^{\prime}, q^{\prime}} & \leqslant K\left\|f_{y}\right\|_{p^{\prime}, q^{\prime}}=K\left[\sum_{\alpha \in J}\left[\int_{I_{\alpha}}|f(x+y)|^{p^{\prime}} d x\right]^{q^{\prime} / p^{\prime}}\right]^{1 / q^{\prime}} \\
& =K|f(y)|\left[\sum_{\alpha \in J}\left[\int_{I_{\alpha}}|f(x)|^{p^{\prime}} d x\right]^{q^{\prime} / p^{\prime}}\right]^{1 / q^{\prime}}=K|f(y)|\|f\|_{p^{\prime}, q^{\prime}}
\end{aligned}
$$


Since $\|f\|_{p^{\prime}, q^{\prime}} \neq 0$, we conclude that $|f(y)| \geqslant 1 / K$ on $G$. This shows that the constant functions belong to $\left(L^{p^{\prime}}, l^{q^{\prime}}\right)$. Since $q>1$, we have $q^{\prime} \neq \infty$, and so we can write

$$
\infty>\|1\|_{p^{\prime}, q^{\prime}}^{q^{\prime}}=\sum_{\alpha \in J}\left[m\left(I_{\alpha}\right)\right]^{q^{\prime} / p^{\prime}}=\sum_{\alpha \in J} m\left(I_{\alpha}\right)=m(G)
$$

Thus $G$ is compact.

4. The case $p<1$. For any nondiscrete group $G$, Żelazko [12] has constructed two functions $f$ and $g$ in $L^{p}(G)$ whose convolution $f * g$ is infinite on a set of positive measure. These functions $f$ and $g$ have compact support and so they belong to $\left(L^{p}, l^{q}\right)(G)$ for any $q>0$. Therefore we have the following theorem.

THEOREM 2. If the amalgam $\left(L^{p}, l^{q}\right)(G), 0<p<1$, is a topological algebra, then $G$ is discrete.

In order to give a converse, we first note that if $G$ is discrete, then $\left(L^{p}, l^{q}\right)(G)=$ $l^{q}(G)$. If $q \leqslant 1$, then $l^{q}(G)$ is a topological algebra [12]. If $q>1$ and $l^{q}(G)$ is an algebra, then $G$ is both discrete and compact, hence finite. And if $G$ is finite, $l^{q}(G)$ is always an algebra. We have thus proved the following corollaries of Theorem 2.

Corollary 1. The amalgam $\left(L^{p}, l^{q}\right)(G), 0<p<1,0<q \leqslant 1$, is a topological algebra if and only if $G$ is discrete.

Corollary 2. The amalgam $\left(L^{p}, l^{q}\right)(G), 0<p<1, q>1$, is a topological algebra if and only if $G$ is finite.

5. The case $p \geqslant 1, q \leqslant 1$. We have seen that for $\left(L^{p}, l^{q}\right)(G)$ to be a topological algebra, $G$ must be compact if $q>1$ and $G$ must be discrete if $p<1$. By contrast with this situation, we show that in the remaining case $(p \geqslant 1, q \leqslant 1)\left(L^{p}, l^{q}\right)(G)$ is always an algebra.

TheOREM 3. The amalgam $\left(L^{p}, l^{q}\right)(G), p \geqslant 1,0<q \leqslant 1$, is a topological algebra under convolution for any locally compact abelian group $G$. Moreover, for $f$ and $g$ in $\left(L^{p}, l^{q}\right)(G)$ we have

$$
\|f * g\|_{p, q} \leqslant 2^{a / q}\|f\|_{p, q}\|g\|_{p, q},
$$

where $a$ is given by the structure theorem as in $\$ 2$.

Proof. We first observe that, in the notation introduced in $\S 2$,

$$
(f * g)(x)=\sum_{\alpha \in J} \sum_{\beta \in J}\left(f_{\alpha} * g_{\beta}\right)(x) .
$$

Therefore, for any $\gamma \in J$, Minkowski's Inequality gives

$$
\begin{aligned}
\left\|(f * g)_{\gamma}\right\|_{p} & =\left\|\sum_{\alpha} \sum_{\beta}\left(f_{\alpha} * g_{\beta}\right)_{\gamma}\right\|_{p} \leqslant \sum_{\alpha} \sum_{\beta}\left\|\left(f_{\alpha} * g_{\beta}\right)_{\gamma}\right\|_{p} \\
& \leqslant \sum_{\substack{\alpha \beta \\
\gamma \subset \alpha+\beta}}\left\|\left(f_{\alpha} * g_{\beta}\right)\right\|_{p},
\end{aligned}
$$


where we use the notation $\gamma \subset \alpha+\beta$ to mean that we sum over all indices $\alpha$ and $\beta$ in $J$ such that $I_{\gamma} \subset I_{\alpha}+I_{\beta}$. Now we apply Young's Inequality for $L^{p}$-spaces to write

$$
\left\|(f * g)_{\gamma}\right\|_{p} \leqslant \sum_{\substack{\alpha \beta \\ \gamma \subset \alpha+\beta}}\left\|f_{\alpha}\right\|_{1}\left\|g_{\beta}\right\|_{p} .
$$

Using the fact that $q \leqslant 1$ together with (2.2), we have

$$
\left\|(f * g)_{\gamma}\right\|_{p}^{q} \leqslant\left(\sum_{\substack{\alpha \beta \\ \gamma \subset \boldsymbol{\alpha}+\beta}}\left\|f_{\alpha}\right\|_{1}\left\|g_{\beta}\right\|_{p}\right)^{q} \leqslant \sum_{\substack{\alpha \beta \\ \gamma \subset \boldsymbol{\alpha}+\beta}}\left\|f_{\alpha}\right\|_{1}^{q}\left\|g_{\beta}\right\|_{p}^{q},
$$

and so

$$
\begin{aligned}
\|f * g\|_{p, q}^{q} & =\sum_{\gamma}\left\|(f * g)_{\gamma}\right\|_{p}^{q}<\sum_{\gamma} \sum_{\alpha} \sum_{\substack{\beta \\
\gamma \subset \alpha+\beta}}\left\|f_{\alpha}\right\|_{1}^{q}\left\|g_{\beta}\right\|_{p}^{q} \\
& =\sum_{\alpha}\left\|f_{\alpha}\right\|_{1}^{q} \sum_{\gamma} \sum_{\substack{\beta \\
\gamma \subset \alpha+\beta}}\left\|g_{\beta}\right\|_{p}^{q} \leqslant 2^{a}\|f\|_{1, q}^{q}\|g\|_{p, q}^{q} .
\end{aligned}
$$

Note the presence of the factor $2^{a}$. This is because, for fixed $\alpha$ and $\gamma$, there are $2^{a} I_{\beta}$ 's such that $I_{\gamma} \subset I_{\alpha}+I_{\beta}$, and as we sum over $\gamma$ each such $\beta$ occurs exactly $2^{a}$ times. Finally, taking $q$ th roots, we obtain

$$
\begin{aligned}
\|f * g\|_{p, q} & \leqslant 2^{a / q}\|f\|_{1, q}\|g\|_{p, q} \\
& \leqslant 2^{a / q}\|f\|_{p, q}\|g\|_{p, q} \quad[\text { by (2.13)]. }
\end{aligned}
$$

This completes the proof.

In view of the fact, established in $\S 2$, that $\left(L^{p}, l^{q}\right)(G)$ is an $F$-space whenever either index is less than 1 , Theorem 3 says that $\left(L^{p}, l^{q}\right)(G), p \geqslant 1, q \leqslant 1$, is an $F$-algebra.

Notice from the proof of Theorem 3 that

$$
\|f * g\|_{p, q} \leqslant 2^{a / q}\|f\|_{1, q}\|g\|_{p, q},
$$

and this shows that $\left(L^{p}, l^{q}\right)(G)$ is an ideal in the algebra $\left(L^{1}, l^{q}\right)(G)$.

\section{REFERENCES}

1. J.-P. Bertrandias, C. Datry and C. Dupuis, Unions et intersections d'éspaces $L^{p}$ invariantes par translation où convolution, Ann. Inst. Fourier (Grenoble) 28 (1978), 53-84.n

2. R. C. Busby and H. A. Smith, Product-convolution operators and mixed-norm spaces, Trans. Amer. Math. Soc. 263 (1981), 309-341.

3. R. R. Goldberg, On a space of functions of Wiener, Duke Math. J. 34 (1967), 683-692.

4. F. Holland, Harmonic analysis on amalgams of $L^{p}$ and $1^{q}$, J. London Math. Soc. (2) 10 (1975), 295-305

5. G. Köthe, Topological vector spaces, Springer-Verlag, New York, 1969.

6. H. Reiter, Classical harmonic analysis and locally compact groups, Oxford Univ. Press, New York and London, 1968

7. W. Rudin, Functional analysis, McGraw-Hill, New York, 1973.

8. J. Stewart, Fourier transforms of unbounded measures, Canad. J. Math. 31 (1979), 1281-1292.

9. K. Urbanik, A proof of a theorem of Żelazko on $L^{p}$-algebras, Colloq. Math. 8 (1961), 121-123. 
10. N. Wiener, On the representation of functions by trigonometric integrals, Math. Z. 24 (1926), 575-616.

11. W. Żelazko, On the algebras $L_{p}$ of locally compact groups, Colloq. Math. 8 (1961), 115-120.

12.

Department of Mathematical Sciences, McMaster University, Hamilton, Ontario, L8S 4K1 Canada

Department of Mathematics, Pennsylvania State University, University Park, Pennsylvania 16802 